\title{
Aggregating risk capital, with an application to operational risk
}

\author{
Paul Embrechts • Giovanni Puccetti
}

Received: 27 September 2005 / Revised: 18 July 2006

(C) Springer Science + Business Media, LLC 2006

\begin{abstract}
We describe a numerical procedure to obtain bounds on the distribution function of a sum of $n$ dependent risks having fixed marginals. With respect to the existing literature, our method provides improved bounds and can be applied also to large non-homogeneous portfolios of risks. As an application, we compute the VaR-based minimum capital requirement for a portfolio of operational risk losses.
\end{abstract}

Keywords Risk aggregation · Dependency bounds · Operational risk · Mass transportation duality theorem $\cdot$ Global optimization

JEL Classification G20 $60 \mathrm{E} 15 \cdot 91 \mathrm{~B} 30$

\section{Introduction}

The Basel Committee on Banking Supervision released a consultative document requiring banks to meet a regulatory capital charge to cover operational risk (OR). The new regulations call for the use of statistical tools to estimate the distribution function of $n$ random variables $X_{1}, \ldots, X_{n}$, representing the OR loss amounts for particular lines/types in a given period. Once the marginal distributions of the $n$ risks are fixed, banks need to measure the risk associated with the aggregate position $S:=X_{1}+\cdots+X_{n}$. With respect to the latter task, the minimum capital requirement is typically calculated as the sum of Value-at-Risks (VaRs) for the estimated marginal distributions at some high level of probability. This standard practice should raise some

\footnotetext{
P. Embrechts

Department of Mathematics, ETH Zurich, CH-8092 Zurich, Switzerland

e-mail: embrechts@math.ethz.ch

G. Puccetti $(\bowtie)$

Department of Mathematics for Decisions, University of Firenze, 50134 Firenze, Italy

e-mail: giovanni.puccetti@dmd.unifi.it
} 
concern, since in general VaR is not a sub-additive measure of risk, and the effect of dependence between the individual losses $X_{i}$ could lead to an under-valuation of the risk associated with the total exposure $S$.

The problem of measuring the VaR of a joint position with fixed marginals is equivalent to the search for the best-possible lower bound on the distribution function of the corresponding aggregate position. Since a full solution to this problem seems to be well out of reach when $n \geq 3$, different bounds have been given in the literature.

So-called standard bounds, obtained from elementary probability, are stated, for instance, in Embrechts and Puccetti [2006, Theorem 3.1]. These bounds are defined as a supremum over $\mathbb{R}^{n-1}$, and very often cannot be handled analytically. Iterative numerical procedures for calculating these bounds are presented in Embrechts et al. [2003] and Denuit et al. [1999]. These techniques, based on the discretization of the marginal distributions, have the disadvantage that their computational complexity increases exponentially in the number $n$ of risks treated. In fact, for non-homogeneous portfolios and apart from the few cases in which an analytical closed form is possible, numerical bounds have not been given in the literature for the sum of more than three risks. Instead of following the approach of the above cited papers, we show that for all continuous marginal models of risk management interest, first-order conditions on that supremum reduce the computation of standard bounds to a simple univariate problem.

Dual bounds, resulting instead from a duality theorem of Rüschendorf [1981], are stated in Embrechts and Puccetti [2006, Theorem 4.2] in the case of an homogeneous portfolio of risks. Since the risk management of OR under the Advanced Measurement Approach requires to deal with different marginal distributions, we extend the above result to heterogeneous portfolios and we describe a numerical procedure to compute the bounds. Dual bounds are shown to be better than the respective standard ones, but, especially in the non-homogeneous scenario, their computation calls for the use of an advanced global optimization algorithm. Moreover, the calculation of standard bounds is still necessary to restrict the feasible region in this optimization to a bounded box in $\mathbb{R}^{n}$.

In Section 2 we give a brief introduction to operational risk and the regulatory framework introduced by the New Basel Capital Accord.

In Section 3 we state the conditions under which the computation of standard bounds turns out to be manageable and give the dual bounds for non-homogeneous portfolios of risks.

In Section 4 we apply the methodologies introduced to a real OR portfolio. Though this is a rather natural real-world application of considerable current interest, the relevance of the probabilistic model studied is much wider and includes aggregation questions in integrated risk management more generally; see for instance Chapter 6 in McNeil et al. [2005]. Another area where aggregation results as discussed in this paper are relevant concerns the calculation of an overall capital charge for banks and/or insurance companies. In this case, capital charges for market, credit and operational risk, respectively underwriting risk, have to be added; see for instance Rosenberg and Schuermann [2006].

A brief appendix concerning extensions to different aggregate positions concludes the paper. 


\section{Operational risk: The Basel II framework}

Under the terms of the New Basel Capital Accord (Basel II) banks will be required to set aside capital for the specific purpose of offsetting operational risk (OR), the risk of losses resulting from inadequate or failed internal processes, people and systems, or external events. In this definition, legal risk is included, but not strategic and reputational risk. A major innovation of the proposed accord is the introduction of an evolutionary framework of three stages for the calculation of an OR capital charge; see Basel Committee on Banking Supervision [2006].

Under the most sophisticated of these approach, namely the Advanced Measurement Approach (AMA), banks are given an unprecedented amount of flexibility to develop their own model for assessing their exposure to OR. This freedom, in the opinion of the Committee, will help to accommodate a rapid evolution in OR management practices over the coming years. Arbitrariness of OR regulatory charges is instead heavily criticized in Pezier [1996]. Nešlehová et al. [2006] contains some critical remarks from a statistical estimation point of view.

The regulator in principle only requires that a bank's activities are categorized into the 56-cell Basel matrix, a two-dimensional array in which operational losses are separately modeled in eight business lines and by seven risk types. The total capital allocation is then requested to be the sum of unspecified risk measures, accounting for expected and unexpected losses for the different OR estimates, and this across the cells of the above or similar matrix. Diversification arguments may be used to reduce this total sum.

\subsection{Probability models for OR losses}

The most risk-sensitive AMA methodology by far is the Loss Distribution Approach (LDA), which aims at modelling the distribution function for $L_{i, j}$, the one-year holding period random OR loss in the $i$-th business line for the $j$-th loss type. Among eligible LDA techniques, the most popular is the actuarial collective model of risk, in which frequency and severity distributions of losses are estimated separately and then the aggregate loss distribution is computed trough convolution over the cells in the Basel matrix. It is typically impossible to derive analytical expressions for this kind of convolution: Monte Carlo simulations or numerical approximation are used in practice; see Frachot et al. [2004] and Hürlimann [2004], respectively. The need for such methods can be bypassed by extracting the underlying one-year loss distribution statistically from available data. This is the approach taken for instance in Moscadelli [2004] and de Fontnouvelle et al. [2005], and critically discussed in Nešlehová et al. [2006]. Alternative approaches may be based on graph theory or econophysics models, possibly combined with some form of expert systems; see Leippold and Vanini [2005] and Kühn and Neu [2004], respectively. A final analysis would typically be based on statistical estimation based on internal as well as external data, combined with expert opinion. Also note that insurance mitigation (up to $20 \%$ ) for operational risk is possible; see for instance Bazzarello et al. [2006]. 


\subsection{Aggregating marginal models}

The Committee proposes to compute the total capital charge $C$ to be allocated by simple addition of the capital charges for every cell of the matrix, regardless of the measure of risk used to calculate them. In standard practice, $C$ is derived as the addition of the VaRs at probability level 0.999 for the random losses $L_{i, j}$ across risk types and business lines, namely

$$
C=\sum_{i=1}^{8} \sum_{j=1}^{7} \operatorname{VaR}_{0.999}\left(L_{i, j}\right),
$$

where $\operatorname{VaR}_{\alpha}\left(L_{i, j}\right), \alpha \in[0,1]$ is the $\alpha$-quantile of the estimated distribution function for the loss in the $(i, j)$-cell of the Basel matrix. This procedure can be mathematically justified by the assumption of comonotonicity among risk cells; see Proposition 3.1 in Embrechts et al. [2003] and the same paper for more details about comonotonicity. Many authors criticize this assumption as not being realistic, and remark that ORs are not perfectly correlated in view of their heterogeneous nature. Among others, Frachot et al. [2004] stress that there are strong arguments in favor of low levels of correlation among aggregate losses in the matrix and regard the one-correlation scenario as highly conservative. At this point we would like to stress that addition of VaRs over aggregated losses, like business lines, is typically done.

In spite of these critics, it seems useful to remark that for many examples of interest in quantitative risk management the maximal value for the VaR for an aggregate position does not occur under the assumption of comonotonicity (i.e. maximal correlation). In fact, apart from pathological cases of no actuarial relevance, for any fixed set of models for the marginal losses $L_{i, j}$ and every quantile $\alpha$ large enough, there always exists a distribution function for the aggregate loss $\sum_{i=1}^{8} \sum_{j=1}^{7} L_{i, j}$ under which

$$
\sum_{i=1}^{8} \sum_{j=1}^{7} \operatorname{VaR}_{\alpha}\left(L_{i, j}\right)<\operatorname{VaR}_{\alpha}\left(\sum_{i=1}^{8} \sum_{j=1}^{7} L_{i, j}\right)
$$

see Embrechts et al. [2005, Section 3.1] and Rüschendorf [1981, Theorem 5 and Pr. 1]. As a consequence, adding VaRs across different business lines turns out not to be a conservative strategy from a pure mathematical point of view. Moreover, the worstVaR structure of dependence can be tricky to deal with, and is often excluded from standard families of copulas used in dependence simulations; see Embrechts et al. [2005, Fig. 3].

The aim of this paper is to compute a conservative estimate of the capital charge $C$ without using any assumption on the dependence structure of the portfolio of risks involved. However, the bank may be permitted to use internally determined models to capture dependence in OR losses; see Chavez-Demoulin et al. [2006] for some advanced approaches to this task. 


\section{Theory}

After giving conditions for an easy calculation of the bound stated in Embrechts and Puccetti [2006, Theorem 3.1], we extend Embrechts and Puccetti [2006, Theorem 4.2] to non-homogeneous portfolios. We first introduce some notation. We usually denote vectors in $\mathbb{R}^{n-1}$ by $\mathbf{x}_{-n}$ and vectors in $\mathbb{R}^{n}$ by $\mathbf{x}$. The indicator function of the set $B \subset \mathbb{R}$ is the function $1_{B}: \mathbb{R} \rightarrow \mathbb{R}$

$$
1_{B}(b):= \begin{cases}1 & \text { if } b \in B \\ 0 & \text { otherwise }\end{cases}
$$

while the inverse of an increasing function $\psi: \mathbb{R} \rightarrow \mathbb{R}$ is the function $\psi^{-1}: \mathbb{R} \rightarrow$ $\overline{\mathbb{R}}:=\mathbb{R} \cup\{+\infty,-\infty\}$, defined by

$$
\psi^{-1}(y):=\inf \{x \in \mathbb{R}: \psi(x) \geq y\}
$$

We denote by $F^{-}$the left-continuous version of the df $F$ of a random variable $X$, i.e. $F^{-}(x):=P[X<x], x \in \mathbb{R}$. Finally, $\mathcal{B M}(\mathbb{R})$ is the class of bounded and measurable real-valued functions.

\subsection{Preliminaries and standard bounds}

Let $X_{1}, \ldots, X_{n}$ be $n(n>1)$ real-valued random variables (risks) on some probability space $(\Omega, \mathfrak{A}, \mathbb{P})$, with given non-defective dfs $F_{i}(x)=\mathbb{P}\left[X_{i} \leq x\right], i \in\{1, \ldots, n\}=$ : $N$. The random vector (portfolio) $\mathbf{X}:=\left(X_{1}, \ldots, X_{n}\right)$ will represent the one-period losses deriving from the $n$ different loss categories of a firm. We assume every marginal distribution $F_{i}, i \in N$ to have support $S_{i}:=\left[a_{i},+\infty\right), a_{i} \in \mathbb{R}$, and to be absolutely continuous with a continuous and strictly decreasing density $f_{i}=F_{i}^{\prime}$ on $\left(\bar{x}_{F_{i}},+\infty\right)$, for some $\bar{x}_{F_{i}} \geq a_{i} \cdot \bar{x}_{F_{i}}$ can be thought as the smallest real number above which the density $f_{i}$ is strictly decreasing. Table 1 gives some important continuous models of interest to risk management, for which the marginal losses are consistent with our framework.

Table 1 Values for $\bar{x}_{F}$ for some distributions $F$ of actuarial interest. We label TGPD the Tail estimator of a Generalized Pareto Distribution; see (6.45) in Embrechts et al. [1997]

\begin{tabular}{lllll}
\hline Distribution & Parameters range & Density & Support & $\bar{x}_{F}$ \\
\hline $\operatorname{Log-Normal}\left(\mu, \sigma^{2}\right)$ & $\mu \in \mathbb{R}, \sigma \geq 0$ & $\frac{1}{x \sqrt{2 \pi} \sigma} e^{-\frac{(\ln (x)-\mu)^{2}}{2 \sigma^{2}}}$ & {$[0,+\infty)$} & $e^{\mu-\sigma^{2}}$ \\
Gamma $(a, b)$ & $a, b>0$ & $\frac{x^{(a-1)} e^{-x / b}}{\Gamma(a) b^{a}}$ & {$[0,+\infty)$} & $b[a-1]^{+}$ \\
Pareto $(\alpha, \lambda)$ & $\alpha, \lambda>0$ & $\frac{\lambda^{\alpha} \alpha}{(\lambda+x)^{\alpha+1}}$ & {$[0,+\infty)$} & 0 \\
Weibull $(\alpha, \lambda)$ & $\alpha, \lambda>0$ & $\left(\frac{x}{\alpha}\right)^{\lambda} \lambda e^{-\left(\frac{x}{\alpha}\right)^{\lambda} x^{-1}}$ & {$[0,+\infty)$} & $\left(\frac{[\lambda-1]^{+}}{\lambda}\right)^{\frac{1}{\lambda}}$ \\
TGPD $\left(\xi, \beta, u, k_{u}\right)$ & $\xi, \beta>0, u \in \mathbb{R}, k_{u} \in(0,1]$ & $\frac{k_{u}}{\beta}\left(1+\frac{\xi(x-u)}{\beta}\right)^{-\frac{1}{\xi}-1}$ & {$[u,+\infty)$} & $u$ \\
\hline
\end{tabular}


The aggregate loss deriving from the portfolio $\mathbf{X}$ will be $S:=\sum_{i=1}^{n} X_{i}$. For a fixed real threshold $s$, we define the function $\phi: \mathbb{R}^{n-1} \rightarrow \mathbb{R}$ as follows:

$$
\phi\left(\mathbf{x}_{-n}\right):=\sum_{i=1}^{n-1} F_{i}\left(x_{i}\right)+F_{n}^{-}\left(s-\sum_{i=1}^{n-1} x_{i}\right)-n+1
$$

The meaning of the function $\phi$ comes out from duality theory; see (3.6) and comments below. In the general case when we do not have information regarding the dependence structure of the portfolio $\mathbf{X}$, e.g. we do not assume the $X_{i}$ 's to be independent or comonotonic, Theorem 3.1 in Embrechts and Puccetti [2006] yields that

$$
\mathbb{P}[S<s] \geq \tau(s):=\sup _{\mathbf{x}_{-n} \in \mathbb{R}^{n-1}}\left[\phi\left(\mathbf{x}_{-n}\right)\right]^{+}
$$

Translated into the language of $\mathrm{VaR}$, the above inequality becomes

$$
\operatorname{VaR}_{\alpha}(S) \leq \tau^{-1}(\alpha), \alpha \in[0,1]
$$

Denuit et al. [1999] define a bound on $\mathbb{P}[S \leq s]$ which is equivalent to $\tau(s)$ in our setting and they remark that it can rarely be calculated explicitly in practice, particularly if the distributions of the risks $X_{i}$ are heterogenous in form. In such circumstances, they resort to a numerical approximation. We instead show that, in our framework of actuarial/financial interest, first-order conditions allow for a less time-consuming search of the supremum in (3.2), even for large portfolios. We let $p:=\max _{i \in N} F_{i}\left(\bar{x}_{F_{i}}\right)$.

Theorem 3.1. If for a fixed real threshold $s, \tau(s)>p$, then $\tau(s)=\phi\left(\mathbf{x}_{-n}^{*}\right)$, where $\mathbf{x}_{-n}^{*}=\left(x_{1}^{*}, \ldots, x_{n-1}^{*}\right)$ is the unique vector in $\prod_{i=1}^{n-1}\left(\bar{x}_{F_{i}},+\infty\right)$ satisfying

$$
\left\{\begin{array}{l}
f_{1}\left(x_{1}^{*}\right)=f_{i}\left(x_{i}^{*}\right), \quad i=2, \ldots, n-1 \\
f_{1}\left(x_{1}^{*}\right)=f_{n}\left(s-\sum_{i=1}^{n-1} x_{i}^{*}\right)
\end{array}\right.
$$

Proof: For $i \in\{1, \ldots, n-1\}$ fixed, observe that

$$
\begin{aligned}
\phi\left(\mathbf{x}_{-n}\right) & =\sum_{\substack{1 \leq j \leq n-1, j \neq i}} F_{j}\left(x_{j}\right)+F_{i}\left(x_{i}\right)+F_{n}^{-}\left(s-\sum_{i=1}^{n-1} x_{i}\right)-n+1 \\
& \leq(n-2)+p+1-n+1=p,
\end{aligned}
$$

for all $\mathbf{x}_{-n}$ with $x_{i} \leq \bar{x}_{F_{i}}$. Considering also that $\tau(s)>p \geq 0$, we can reduce problem (3.2) to

$$
\tau(s)=\sup _{\mathbf{x}_{-n} \in \mathbb{R}^{n-1}}\left[\phi\left(\mathbf{x}_{-n}\right)\right]^{+}=\sup _{\mathbf{x}_{-n} \in \mathbb{R}^{n-1}} \phi\left(\mathbf{x}_{-n}\right)=\sup _{\mathbf{x}_{-n} \in \prod_{i=1}^{n-1}\left(x_{F_{i}},+\infty\right)} \phi\left(\mathbf{x}_{-n}\right) .
$$


Note that

$$
\lim _{x_{i} \rightarrow+\infty} F_{n}^{-}\left(s-x_{i}-\sum_{\substack{1 \leq j \leq n-1, j \neq i}} \bar{x}_{F_{j}}\right)=0, \quad \text { for all } i=1, \ldots, n-1 .
$$

Therefore, for all $\xi>0$ and $i \in\{1, \ldots, n-1\}$, it is always possible to select a real $x_{i}^{+}$such that

$$
F_{n}^{-}\left(s-x_{i}-\sum_{\substack{1 \leq j \leq n-1, j \neq i}} \bar{x}_{F_{j}}\right)<\xi \text {, for all } x_{i} \geq x_{i}^{+}
$$

This implies that

$$
\begin{aligned}
\phi\left(\mathbf{x}_{-n}\right) & =\sum_{i=1}^{n-1} F_{i}\left(x_{i}\right)+F_{n}^{-}\left(s-\sum_{i=1}^{n-1} x_{i}\right)-n+1 \\
& \leq(n-1)+\xi-n+1=\xi,
\end{aligned}
$$

for all $\mathbf{x}_{-n} \in \prod_{i=1}^{n-1}\left(\bar{x}_{F_{i}},+\infty\right)$ with $x_{i} \geq x_{i}^{+}$.

We can choose $\xi$ such that $0 \leq p<\xi<\tau(s)$ and conclude that $\bar{x}_{F_{i}}<x_{i}^{+}, i=$ $1, \ldots, n-1$ and hence there exists $\mathbf{x}_{-n}^{*} \in \prod_{i=1}^{n-1}\left(\bar{x}_{F_{i}}, x_{i}^{+}\right)$such that $\tau(s)=\phi\left(\mathbf{x}_{-n}^{*}\right)$.

The continuity of the $f_{i}$ 's yields differentiability of $\phi$ on $\prod_{i=1}^{n-1}\left(\bar{x}_{F_{i}},+\infty\right)$, implying that $\mathbf{x}_{-n}^{*}$ must satisfy the first-order conditions given in (3.4). Moreover, note that $\left(s-\sum_{i=1}^{n-1} x_{i}^{*}\right)>\bar{x}_{F_{n}}$, otherwise $\phi\left(\mathbf{x}_{-n}^{*}\right) \leq n-1+F_{n}^{-}\left(\bar{x}_{F_{n}}\right)-n+1 \leq p$. As a consequence, strict decreasingness of each $f_{i}$ in $\left(\bar{x}_{F_{i}},+\infty\right)$ gives uniqueness of such a solution. Note that, for $n=2,(3.4)$ reduces to its second equality.

Remark 3.1. Though the bound (3.2) is valid for any set of marginal distributions $F_{1}, \ldots, F_{n}$, conditions (3.4) may fail to hold at the corresponding supremum if some of the $F_{i}$ 's have a bounded support or are mixed with a discrete component in the tail, as for instance in a compound model. For a in-depth discussion on the assumption of continuous marginals and on the way actuarial models fit our framework, we refer to Section 5.1. For a general joint loss $\psi(\mathbf{X})$ first-order conditions are still necessary at the global optimum but may fail to be sufficient; see Appendix A.

We can calculate bounds on $\operatorname{VaR}_{\alpha}(S)$ using Theorem 3.1 only for values $\alpha>p$. This does not present an issue, since, in practice, $\alpha$ is often chosen above the level 0.99 . The corresponding thresholds of interest are hence sufficiently large. 
Lemma 3.1. We have that $\tau(s)>$ for every real threshold $s$ satisfying

$$
s>\sum_{i=1}^{n} F_{i}^{-1}\left(\frac{p+n-1}{n}\right)
$$

Proof: Assume that (3.5) is true and define the vector $\hat{\mathbf{x}}$ as $\hat{x}_{i}:=F_{i}^{-1}\left(\frac{p+n-1}{n}\right), i \in$ $N$. Since every $f_{i}$ is positive and $\frac{p+n-1}{n}>p$, we conclude that $F_{n}^{-}\left(s-\sum_{i=1}^{n-1} \hat{x}_{i}\right)>$ $F_{n}^{-}\left(F_{n}^{-1}\left(\frac{p+n-1}{n}\right)\right)=\frac{p+n-1}{n}$ and therefore

$$
\tau(s)=\sup _{\mathbf{x}_{-n} \in \mathbb{R}^{n-1}}\left[\phi\left(x_{-n}\right)\right]^{+} \geq\left[\phi\left(\hat{x}_{-n}\right)\right]^{+}>n\left(\frac{p+n-1}{n}\right)-n+1=p .
$$

Remark 3.2. For an homogeneous portfolio, i.e. $F_{i}=F, i \in N$ for some fixed df $F$, we have $p=F\left(\bar{x}_{F}\right)$ and the standard bound simplifies to

$$
\tau(s)=n F(s / n)-n+1, s \geq n F^{-1}\left(\frac{F\left(\bar{x}_{F}\right)+n-1}{n}\right) ;
$$

see Embrechts and Puccetti [2006]. Moreover, note that if $p=0$, e.g. in the case of a portfolio of Pareto-distributed risks, conditions (3.4) can be used for every real threshold yielding a non-trivial standard bound.

Recall from (3.3) that the inverse of the function $\tau$ gives a bound on the VaR for the aggregate position $S$. The function $\tau^{-1}$ can be directly calculated using Theorem 4.1 in Embrechts et al. [2003], which is the dual formulation of the optimization problem in (3.2). Using this result, we have analogous interior first-order conditions; for further details in the case of homogeneous portfolios, we refer to Theorem 2.6 in Puccetti [2005], the general case being analogous. We do not follow this equivalent approach here, since we will need the vector $\mathbf{x}_{-n}^{*}$ in the analysis below.

\subsection{Dual bounds}

For portfolios of more than two risks ( $n>2)$, the standard bound given in (3.2) is not sharp in general; see Embrechts and Puccetti [2006] and discussions therein. A way to tighten this bound relies on a Mass Transportation duality result given in Rüschendorf [1981, Theorem 5]:

$$
\begin{aligned}
\mathbb{P}[S<s] \geq & 1-\inf \left\{\sum_{i=1}^{n} \int f_{i} d F_{i}: f_{i} \in \mathcal{B M}(\mathbb{R}), i \in N\right. \text { s.t. } \\
& \left.\sum_{i=1}^{n} f_{i}\left(x_{i}\right) \geq 1_{[s,+\infty)}\left(\sum_{i=1}^{n} x_{i}\right) \text { for all } x_{i} \in \mathbb{R}, i \in N\right\} .
\end{aligned}
$$


This dual optimization problem seems to be very hard to solve in general, but every set of admissible functions $\left(f_{1}, \ldots, f_{n}\right)$ in (3.6) produces a lower bound $\alpha$ on $\mathbb{P}[S<s]$, and, equivalently, an upper bound on $\operatorname{VaR}_{\alpha}(S)$. In general, choosing in (3.6) the piecewise-constant admissible dual choices

$$
f_{i}(x):=1_{\left(x_{i},+\infty\right)}(x), i \in N \backslash\{n\}, f_{n}(x):=1_{\left[s-\sum_{i=1}^{n-1} x_{i},+\infty\right)}(x),
$$

for arbitrary $\mathbf{x}_{-n} \in \mathbb{R}^{n-1}$ and $s \in \mathbb{R}$, leads to the function $\phi$ defined in (3.1) and thus to the standard bound $\tau(s)$. We now consider piecewise-linear admissible choices in order to improve the latter.

The following theorem extends Theorem 4.2 in Embrechts and Puccetti [2006] to heterogeneous portfolios. Recall that $\mathbf{x}_{-n}^{*}$ is the unique vector in $\prod_{i=1}^{n-1}\left(\bar{x}_{F_{i}},+\infty\right)$ satisfying (3.4).

Theorem 3.2. Define the vector $\mathbf{x}^{*}:=\left(\mathbf{x}_{-n}^{*}, s-\sum_{i=1}^{n-1} x_{i}^{*}\right)$. Then

$$
\mathbb{P}[S<s] \geq \delta(s):=1-\inf _{\mathbf{r} \in \prod_{i=1}^{n}\left(a_{i}, x_{i}^{*}\right)} \frac{\sum_{i=1}^{n} \int_{r_{i}}^{s-\sum_{j \neq i} r_{j}}\left(1-F_{i}\left(x_{i}\right)\right) d x_{i}}{s-\sum_{i=1}^{n} r_{i}} .
$$

Translated into the language of $\mathrm{VaR}$, the above inequality becomes

$$
\operatorname{VaR}_{\alpha}(S) \leq \delta^{-1}(\alpha), \alpha \in[0,1]
$$

Proof: For an arbitrary vector $\mathbf{r} \in \prod_{i=1}^{n}\left(a_{i}, x_{i}^{*}\right)$ define, for all $i \in N$, the functions $\hat{f}_{i}^{\mathbf{r}}: \mathbb{R} \rightarrow \mathbb{R}$

$$
f_{i}^{\mathbf{r}}\left(x_{i}\right):= \begin{cases}0 & \text { if } x_{i} \leq r_{i} \\ \frac{x_{i}-r_{i}}{s-\sum_{i=1}^{n} r_{i}} & \text { if } r_{i}<x_{i} \leq s-\sum_{j \neq i} r_{j} \\ 1 & \text { otherwise. }\end{cases}
$$

We now prove that $\hat{f}_{1}^{\mathbf{r}}, \ldots, \hat{f}_{n}^{\mathbf{r}}$ are an admissible choice in (3.6). Since every $\hat{f}_{i}^{\mathbf{r}}$ is nonnegative, it is sufficient to show that we have $\sum_{i=1}^{n} \hat{f}_{i}^{\mathbf{r}}\left(x_{i}\right) \geq 1$ whenever $\sum_{i=1}^{n} x_{i} \geq s$. If $x_{\hat{i}} \geq s-\sum_{j \neq i} r_{j}$ for some $\hat{i}=1, \ldots, n$, this trivially follows, so take $x_{i} \leq s-$ $\sum_{j \neq i} r_{j}, i \in N$ with $\sum_{i=1}^{n} x_{i} \geq s$. Define

$$
I:=\left\{i \in N: x_{i}>r_{i}\right\}, \quad \bar{I}:=N \backslash I
$$

and observe that

$$
\sum_{i \in I} x_{i} \geq s-\sum_{i \in \bar{I}} x_{i} \geq s-\sum_{i \in \bar{I}} r_{i}
$$


By definition of the $\hat{f}_{i}^{\mathrm{r}}$ 's and (3.8), it follows that

$$
\begin{aligned}
\sum_{i=1}^{n} \hat{f}_{i}^{\mathbf{r}}\left(x_{i}\right)=\sum_{i \in I} \hat{f}_{i}^{\mathbf{r}}\left(x_{i}\right)=\sum_{i \in I} \frac{x_{i}-r_{i}}{s-\sum_{i=1}^{n} r_{i}} & =\frac{\sum_{i \in I} x_{i}-\sum_{i \in I} r_{i}}{s-\sum_{i=1}^{n} r_{i}} \\
& \geq \frac{s-\sum_{i \in \bar{I}} r_{i}-\sum_{i \in I} r_{i}}{s-\sum_{i=1}^{n} r_{i}} \geq 1 .
\end{aligned}
$$

The theorem follows by checking that

$$
\sum_{i=1}^{n} \int \hat{f}_{i}^{\mathbf{r}}\left(x_{i}\right) d F_{i}\left(x_{i}\right)=\frac{\sum_{i=1}^{n} \int_{r_{i}}^{s-\sum_{j \neq i} r_{j}}\left(1-F_{i}\left(x_{i}\right)\right) d x_{i}}{s-\sum_{i=1}^{n} r_{i}}
$$

and taking the infimum over all $\mathbf{r} \in \prod_{i=1}^{n}\left(a_{i}, x_{i}^{*}\right)$.

Remark 3.3. If $\mathbf{r}$ goes to $\mathbf{x}^{*}$, we have that, for all $i \in N, \hat{f}_{i}^{\mathbf{r}}$ goes in the sup-norm to the function $\bar{f}_{i}: \mathbb{R} \rightarrow \mathbb{R}, \bar{f}_{i}:=1_{\left(x_{i}^{*},+\infty\right)}\left(x_{i}\right)$. Since substituting these admissible choices in (3.7) we find that $\delta(s)=\tau(s)$, the dual bound $\delta$ is in general better (i.e. $\geq$ ) than the standard bound $\tau$. This can be equivalently expressed by saying that

$$
\operatorname{VaR}_{\alpha}(S) \leq \delta^{-1}(\alpha) \leq \tau^{-1}(\alpha), \alpha \in[0,1]
$$

Moreover, note that every choice for the vector $\mathbf{r}$, even if not optimal, provides a bound on the corresponding VaR for $S$, which is conservative from a risk management point of view.

\section{Applications to Operational Risk data}

Our aim here is to illustrate a general procedure that, once the univariate marginals $F_{1}, \ldots, F_{n}$ of a portfolio of risks are fixed, will allow to calculate the standard and dual bounds defined respectively in (3.2) and (3.7).

\subsection{Data from the second QIS}

Moscadelli [2004] contains an analysis of the Basel II data on Operational Risk coming out of the second Quantitative Impact Study (QIS); see also Chapter 10 in McNeil et al. [2005] for a discussion and further references. As an illustration of our results, we choose as marginals the distribution functions estimated in the paper of Moscadelli [2004] for the losses in eight OR business lines. We thus take every marginal risk $X_{i}, i=1, \ldots, 8$ to be distributed as the Tail estimator of a Generalized Pareto Distribution (TGPD), i.e.

$$
F_{i}(x)=1-k_{u_{i}}\left(1+\frac{\xi_{i}\left(x-u_{i}\right)}{\beta_{i}}\right)^{-\frac{1}{\xi_{i}}}, x>u_{i}, \quad \text { for all } i=1, \ldots, 8 .
$$


Table 2 Parameter values for the eight TGPD-distributed risks following Moscadelli [2004]

\begin{tabular}{lllllll}
\hline Business line & $i$ & $\xi_{i}$ & $\beta_{i}$ & $u_{i}$ & $k_{u_{i}}$ & $F\left(u_{i}\right)$ \\
\hline Corporate finance & 1 & 1.19 & 774 & 400.28 & 0.09929 & 0.90071 \\
Trading \& sales & 2 & 1.17 & 254 & 193.00 & 0.09977 & 0.90023 \\
Retail banking & 3 & 1.01 & 233 & 247.00 & 0.03462 & 0.96538 \\
Commercial banking & 4 & 1.39 & 412 & 270.00 & 0.09227 & 0.90773 \\
Payment \& settlement & 5 & 1.23 & 107 & 110.00 & 0.10097 & 0.89903 \\
Agency services & 6 & 1.22 & 243 & 201.66 & 0.10604 & 0.89396 \\
Asset management & 7 & 0.85 & 314 & 235.00 & 0.09648 & 0.90352 \\
Retail brokerage & 8 & 0.98 & 124 & 149.51 & 0.09979 & 0.90021 \\
\hline
\end{tabular}

The values for the parameters in the different business lines are chosen in accordance with the above cited paper and are summarized in Table 2.

The reasons for that choice are mainly pedagogical: we are not primarily interested here in the many remaining statistical problems (lack of extreme data, reporting bias, limited collection periods, cut-off values, etc...) typically present in the analysis of OR data, for which we refer to Frachot et al. [2004], de Fontnouvelle et al. [2005] and to Section 5 below. The reader may notice that we deal with 8 categories of OR losses, instead of the 56 in the full Basel matrix. This is done in view of computational issues; see Section 4.3 below.

\subsection{Calculation of standard bounds}

The above portfolio of OR risks fits our framework with $a_{i}=\bar{x}_{F_{i}}=u_{i}$, for all $i=$ $1, \ldots, 8$; see Table 1 . We point out that our setting is suitable also in those situations where one only has an accurate modeling of the extreme right part of the marginal distributions.

In order to calculate the standard bound $\tau(s)$, we can use the conditions stated in Theorem 3.1 provided that $\tau(s)>\max _{i=1, \ldots, 8} F_{i}\left(u_{i}\right)=0.96538$; see Table 2 . According to Lemma 3.1, this condition is met if the threshold $s$ is set above the value $7.9917 \times 10^{4}$. If this is the case, conditions (3.4) are necessary and sufficient to identify the vector $\mathbf{x}_{-n}^{*}$ attaining the standard bound. For a portfolio of TGPD marginals, $\mathbf{x}_{-n}^{*}=\left(x_{1}^{*}, \ldots, x_{n-1}^{*}\right)$ must satisfy:

$$
\begin{aligned}
& \frac{k_{u_{1}}}{\beta_{1}}\left(1+\frac{\xi_{1}\left(x_{1}^{*}-u_{1}\right)}{\beta_{1}}\right)^{-\frac{1}{\xi_{1}}-1}=\frac{k_{u_{i}}}{\beta_{i}}\left(1+\frac{\xi_{i}\left(x_{i}^{*}-u_{i}\right)}{\beta_{i}}\right)^{-\frac{1}{\xi_{i}}-1}, \quad i=2, \ldots, n-1, \\
& \frac{k_{u_{1}}}{\beta_{1}}\left(1+\frac{\xi_{1}\left(x_{1}^{*}-u_{1}\right)}{\beta_{1}}\right)^{-\frac{1}{\xi_{1}}-1}=\frac{k_{u_{n}}}{\beta_{n}}\left(1+\frac{\xi_{n}\left(s-\sum_{i=1}^{n-1} x_{i}^{*}-u_{n}\right)}{\beta_{n}}\right)^{-\frac{1}{\xi_{n}}-1}
\end{aligned}
$$

It is numerically straightforward to find the unique vector solving these equations. In fact, the values for $x_{2}^{*}, \ldots, x_{n-1}^{*}$ are uniquely determined by $x_{1}^{*}$ via (4.1), while $x_{1}^{*}$ is 
the unique root of the function $\zeta: \mathbb{R} \rightarrow \mathbb{R}$, defined as follows:

$$
\begin{aligned}
\zeta\left(x_{1}\right):= & \frac{k_{u_{1}}}{\beta_{1}}\left(1+\frac{\xi_{1}\left(x_{1}-u_{1}\right)}{\beta_{1}}\right)^{-\frac{1}{\xi_{1}}-1} \\
& -\frac{k_{u_{n}}}{\beta_{n}}\left(1+\frac{\xi_{n}\left(s-\sum_{i=1}^{n-1} x_{i}^{*}\left(x_{1}\right)-u_{n}\right)}{\beta_{n}}\right)^{-\frac{1}{\xi_{n}}-1},
\end{aligned}
$$

with

$$
\begin{gathered}
x_{i}^{*}\left(x_{1}\right):=u_{i}+\frac{\beta_{i}}{\xi_{i}}\left\{\left[\frac{\beta_{i}}{\beta_{1}} \frac{k_{u_{1}}}{k_{u_{i}}}\left(1+\frac{\xi_{1}\left(x_{1}-u_{1}\right)}{\beta_{1}}\right)^{-\frac{1}{\xi_{1}}-1}\right]^{-\frac{\xi_{i}}{1+\xi_{i}}}-1\right\}, \\
i=2, \ldots, n-1 .
\end{gathered}
$$

Finding the unique root of a real-valued function is a standard routine for most mathematical software. We use MATLAB ${ }^{\circledR}$ to obtain the vector $\mathbf{x}_{-n}^{*}$ and the corresponding standard bound for the thresholds illustrated in Fig. 1. The corresponding bounds on VaR are given in Table 3.

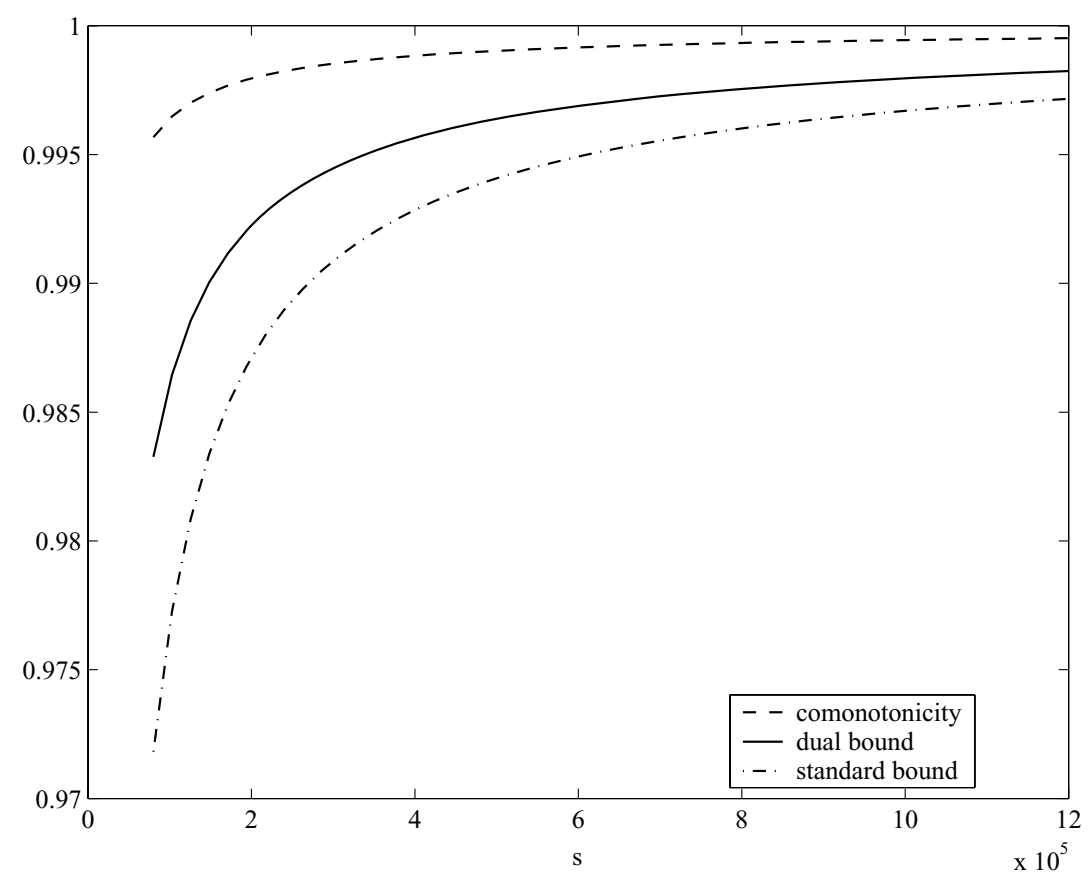

Fig. 1 Range for $\mathbb{P}[S<s]$ for Moscadelli's portfolio of risks under the comonotonic scenario. We also give the standard (3.2) and dual (3.7) bounds 
Table 3 Range for $\operatorname{VaR}_{\alpha}\left(\sum_{i=1}^{8} X_{i}\right)$ for the data underlying Table 2

\begin{tabular}{llll}
\hline$\alpha$ & Comonotonic value & Dual bound & Standard bound \\
\hline 0.99 & $2.8924 \times 10^{4}$ & $1.4778 \times 10^{5}$ & $2.6950 \times 10^{5}$ \\
0.995 & $6.7034 \times 10^{4}$ & $3.3922 \times 10^{5}$ & $6.1114 \times 10^{5}$ \\
0.999 & $4.8347 \times 10^{5}$ & $2.3807 \times 10^{6}$ & $4.1685 \times 10^{6}$ \\
0.9999 & $8.7476 \times 10^{6}$ & $4.0740 \times 10^{7}$ & $6.7936 \times 10^{7}$ \\
\hline
\end{tabular}

\subsection{Computation of dual bounds}

While the computation of standard bounds is reduced to the problem of finding the root of a real-valued function, calculating the dual bounds calls for the use of sophisticated optimization algorithms. In fact, problem (3.7) introduces the possibility of multiple local-optima, i.e. spurious solutions that merely satisfy the requirements on the partial derivatives of the objective function. Problems exhibiting the above difficulty and algorithms designed to tackle it are termed Global Optimization (GO) problems. In particular, (3.7) is a bound-constrained GO problem, i.e. having a bounded box as a feasible region in $\mathbb{R}^{n}$.

Actually, there are several optimization techniques fitting the structure of (3.7). We refer to Neumaier [2004] for a comprehensive review. We only remark that it is unrealistic to expect to find one general non-linear algorithm working well for every kind of non-linear model. As a consequence, GO codes are to be used with care: they cannot always find the global optimum in a reasonable, predictable amount of time and their quality of performance is not well understood. Moreover, algorithmic performance typically deteriorates with increasing dimension. Choosing $n=50$ is challenging for most general-purpose codes dealing with heterogeneous portfolios; that is why we did not consider all the 56 cells of the Basel Matrix in our application. With respect to this, note instead that, for identically distributed risks, problem (3.7) is trivial to solve even with huge portfolio dimensions; see Puccetti [2005, Table 2.4].

Our present understanding of GO codes implies that one should experiment with several methods. Keeping an eye at the industry needs, among all the codes we tried yielding satisfactory results, we decided for freely-available softwares which have a MATLAB ${ }^{\circledR}$ version and are well documented.

Since loops and conditional statements are executed in $\mathrm{C}$ much faster than in MATLAB $^{\circledR}$, one should expect the $C$ implementation of the same algorithms to dramatically reduce the computational time. On the other hand, the largest manageable portfolio dimension is expected to increase when using specialized software which require the gradient and Hessian matrix of the objective function.

For these more sophisticated choices or latest advances on GO software to be possibly used, we refer to the regularly updated Fourer [2000] and Neumaier [2000], and to the references cited therein.

\subsection{Optimization codes}

In order to solve problem (3.7) and find $\delta(s)$ for the data underlying Table 2, we use two similar codes which are known in the literature to be effective global optimizers that require relatively few function evaluations: 
(i) Global optimization by Multilevel Coordinate Search (MCS), developed by Huyer and Neumaier [1999]; MATLAB ${ }^{\circledR}$ version and user guide are available at http: / / www. mat. univie.ac.at/ neum/software/mcs/

(ii) DIviding RECTangles (DIRECT), originally introduced in Jones et al. [1993]; MATLAB $^{\circledR}$ version and user guide are available at http://www4.ncsu.edu/ $\sim_{\text {Ctk/Finkel_Direct/ }}$

For more details on the structure of the two algorithms and the underlying theory, we refer the reader to the above cited papers.

Both methods are guaranteed to converge eventually to the globally optimal function value provided that the function is continuous in the neighborhood of a global optimizer. Unfortunately, this eventually may come at the expense of a large and exhastive search over the domain; see for instance Huyer and Neumaier [1999, Section 6]. In fact, the two codes are so-called sampling algorithms, i.e. they sample function values on the domain and need a criterion that tells the program when to stop searching for a better local minimizer. This criterion should be stringent enough that it will not waste too many functions values, but it should also be sufficiently flexible to ensure that in typical cases the algorithm does not terminate before the global minimizer has been found. We stress that, even in the latter case, the corresponding dual bound will be conservative; see Remark 3.3. Finally, the two codes are deterministic, hence no multiple runs are needed.

By applying the two codes to the objective function given in (3.7) with the marginal distributions from Table 2, we obtain the same results, which are illustrated in Fig. 1 and Table 3 along with the previously calculated standard bounds. It is relevant that in Fig. 1 the dual bound is strictly greater than the standard one, in accordance with Remark 3.3. Observe also that the value for $\mathbb{P}[S<s]$ obtained under the assumption of comonotonicity among risks lies well above the corresponding dual bound defined in (3.7), and this for all thresholds $s$ of interest. As we already stated in (2.1), the sharp bound on $\mathbb{P}[S<s]$ falls between the comonotonic and the dual values, highlighting again that VaR is not a sub-additive measure of risk. This is also evident from Table 3, where the corresponding bounds on VaR are given. The comonotonic value is obtained by simple summation of marginal VaRs across business lines.

We stress the gain an end user may have in evaluating the risk of a portfolio by the the dual rather than the standard bound. Indeed, the lowest bound offers an evaluation of the risky position held that is prudential, more realistic and economically advantageous at the same time. Though Frachot et al. [2004] among others consider even the comonotonic charges as over-conservative, there is no mathematical reason to drop the worst-case bounds if one uses VaR to evaluate the risk of the position held and no dependence assumptions on the portfolio are explicitly made.

As a further pedagogical example, we apply our procedure to the three-dimensional Pareto-Pareto-Weibull portfolio described in Table 2 in Denuit et al. [1999]. In this paper, the authors resort to the numerical approximation of the marginal distributions described in Williamson and Downs [1990], in order to compute the standard bound $F_{\min }$, which, in our setting, is equivalent to the standard bound $\tau$. Table 4 collects the standard bounds $F_{\min }(s)$ calculated in the above paper along with the standard bounds $\tau(s)$ and the dual bounds $\delta(s)$, calculated using Theorem 3.1 and Theorem 3.2, respectively. Note that, apart from rounding errors, our standard bounds $\tau(s)$ are exact Springer 
Table 4 Lower bounds on $\mathbb{P}\left[X_{1}+X_{2}+X_{3}<s\right]$ for the portfolio of risks described in Denuit et al. [1999, Table 2]

\begin{tabular}{llllllll}
\hline$s$ & $F_{\min }(s)$ & $\tau(s)$ & $\delta(s)$ & $s$ & $F_{\min }(s)$ & $\tau(s)$ & $\delta(s)$ \\
\hline 2.75 & 0.052 & 0.0535 & 0.1650 & 6.50 & 0.652 & 0.6528 & 0.6961 \\
3.00 & 0.124 & 0.1254 & 0.2294 & 6.75 & 0.668 & 0.6719 & 0.7128 \\
3.25 & 0.188 & 0.1900 & 0.2872 & 7.00 & 0.688 & 0.6896 & 0.7282 \\
3.50 & 0.248 & 0.2482 & 0.3391 & 7.25 & 0.704 & 0.7060 & 0.7427 \\
3.75 & 0.300 & 0.3010 & 0.3860 & 7.50 & 0.720 & 0.7214 & 0.7561 \\
4.00 & 0.348 & 0.3489 & 0.4284 & 7.75 & 0.732 & 0.7357 & 0.7686 \\
4.25 & 0.392 & 0.3924 & 0.4669 & 8.00 & 0.748 & 0.7490 & 0.7803 \\
4.50 & 0.432 & 0.4321 & 0.5020 & 8.25 & 0.760 & 0.7616 & 0.7913 \\
4.75 & 0.468 & 0.4684 & 0.5340 & 8.50 & 0.772 & 0.7733 & 0.8015 \\
5.00 & 0.500 & 0.5017 & 0.5633 & 8.75 & 0.784 & 0.7843 & 0.8111 \\
5.25 & 0.532 & 0.5322 & 0.5902 & 9.00 & 0.792 & 0.7946 & 0.8201 \\
5.50 & 0.560 & 0.5604 & 0.6149 & 9.25 & 0.804 & 0.8042 & 0.8286 \\
5.75 & 0.584 & 0.5863 & 0.6377 & 9.50 & 0.812 & 0.8133 & 0.8365 \\
6.00 & 0.608 & 0.6102 & 0.6587 & 9.75 & 0.820 & 0.8219 & 0.8440 \\
6.25 & 0.632 & 0.6323 & 0.6781 & 10.00 & 0.828 & 0.8300 & 0.8511 \\
\hline
\end{tabular}

since they are not based on some discretization of the marginal distributions. Indeed, they are greater than the corresponding $F_{\min }(s)$, according to Denuit et al. [1999, Section 4]. Moreover, the dual bounds $\delta(s)$ improve the corresponding standard bounds without using additional information on the dependence structure of the portfolio.

\section{Final remarks}

This paper describes a procedure to calculate standard bounds on the distribution of an aggregate loss with given marginals which is more accurate than the iterative methods existing in the literature. Most importantly, the new technique can be applied when the number $n$ of risks involved is large. This is because our method always reduces to the search for the unique root of a real-valued function.

A dual approach from Mass Transportation theory allows to improve the standard bound so obtained when the latter is not sharp ( $n \geq 3)$, but calls for the use of a Global Optimization algorithm and may come at the expence of a large computational time for medium-sized portfolios $(10 \leq n \leq 50)$. The time consumption is typically reduced if all marginal distributions come from the same parameterized family, as in the operational risk example given in Section 4. A general procedure to calculate standard and dual bounds on the distribution function of the total loss originating from a general portfolio of risks goes as follows:

(i) Substitute the fixed marginal densities in (3.4) and determine the real function $\zeta$ whose unique root is the first component of the vector $\mathbf{x}_{-n}^{*}$ which attains the standard bound (3.2).

(ii) Substitute the tails of the marginal distributions and the vector $\mathbf{x}^{*}$ (defined from $\mathbf{x}_{-n}^{*}$ ) in (3.7) and apply a GO algorithm to solve the bound-constrained problem so obtained. The optimum function value gives the dual bound (3.7). 
If the mathematical form of the densities does not allow to write the function $\zeta$ in closed form, the GO codes described in Section 4.4 may become useful also to determine the standard bounds. However, an analytical expression is always to be preferred.

\subsection{Practical challenges: Actuarial collective models}

Our standard-dual bound methodology relies on the assumption of continuity at least in the tail of the distributions $F_{1}, \ldots, F_{n}$ of the $n$ one-period losses considered.

In practice, banks rarely have enough available data for calibrating these probability distributions. On the contrary, banks frequently have to provide an assessment of their frequency and severity parameters on the basis of both their own experts' point of view and available data (internal as well as external) if any. This means that actuarial collective models are often used throughout the banking industry as the most popular way of approximating the $F_{i}$ 's. In the context of actuarial models, the techniques proposed in this paper are difficult to implement, first because marginal distributions have no analytical expressions and secondly because of the compounding nature of these distributions.

In order to adapt our mathematical methods to collective models, it is useful to recall that any compound model with sub-exponential severities of claims can be approximated in the tail by a continuous distribution, see Embrechts et al. [1997, Theorem 1.3.9].

Concerning the AMA methodology, we agree with the author of Moscadelli [2004] in saying that the treatment of the severity and frequency components within a single overall estimation problem may reduce the estimation error and the computational cost with respect to compound models. Further support to continuous models comes from statistical analyses, which show that Extreme Value Theory in its Peaks Over Thresholds representation explains the behavior of OR data very well at the highest percentiles, while conventional actuarial models are typically underperforming in the tail area of data. In particular, the use of TGPD distributions in modeling OR losses has a strong mathematical justification; see Theorem 3.4.13(b) in Embrechts et al. [1997]. For further background on the use of these techniques to OR data, and in particular for a warning concerning data contamination and mixture models, see Nešlehová et al. [2006].

In conclusion, the usual lack of data in the extreme right part of the loss distributions, combined with the necessity of high-quantile estimates, calls for the use of a smooth parameterized model for the tail of losses.

\subsection{Inclusion of jumps in the model}

Real world catastrophes are often characterized by extraordinary movements that could be included as discontinuities or jumps for added realism in general risk management models. We now briefly discuss how the inclusion of jumps would affect the results in this paper.

Finding the worst-possible VaR for a joint position with given discrete marginals is a matter of solving a linear problem (LP) using the standard techniques from operations research. Unfortunately, the dimensions of that LP increase exponentially with the number of risks in the portfolio of interest. In practice, even for small-sized portfolios 
$(n=5)$, the need for less computationally-demanding methods arises; see Section 5.1 in Embrechts and Puccetti [2006] for more details on this.

With respect to standard bounds, when introducing discontinuities in the marginal densities $f_{1}, \ldots, f_{n}$ of the $n$ one-period losses, the first-order conditions introduced in (3.4) may fail to determine a vector $\mathbf{x}_{-n}^{*}$ attaining the supremum in (3.2). If this is the case, the most efficient technique to calculate standard bounds is the one first introduced in Williamson and Downs [1990] for portfolios of two marginals and then extended to arbitrary portfolios in Denuit et al. [1999] and in Embrechts et al. [2003]. This latter procedure however does not provide the vector $\mathbf{x}_{-n}^{*}$, which is instead needed for the computation of the corresponding dual bound.

In order to find such a vector one has to find the solution of (3.2). In the case of discrete marginal, this turns out to be a global optimization problem with an objective function taking only a finite number of values. An efficient code for this purpose can be looked for in Fourer [2000] and Neumaier [2000].

Finally, dual bounds are based on the admissibility of the dual functionals $\hat{f}_{1}^{\mathbf{r}}, \ldots, \hat{f_{n}^{\mathbf{r}}}$ defined in the proof of Theorem 3.2, and hence are formally independent of the choice of the $F_{i}$ 's. However, an end user working with some particular discrete distribution functions may find it useful to construct ad-hoc admissible dual choices yielding a very good bound within the specific context.

When using mixed distributions (as those in compound models), things get much more complicated. For example, the search for the vector $\mathbf{x}_{-n}^{*}$ giving the supremum in (3.2), to be performed over a continuum of points and function values without even a bounded range, may result very hard to carry out. In this case, as remarked in Section 5.1 above, we recommend to use continuous interpolations of the tail of the distributions of interest.

\section{Appendix A Extensions to general aggregating functions}

In this appendix we discuss an extension to the case in which the total exposure deriving from the $n$ marginal risks is a general increasing function of these marginal risks. Typical situations where this may occur are to be found in credit risk (basket options) and multi-line insurance or reinsurance. For examples, see Chapter 6 in McNeil et al. [2005].

\section{A.1 Standard bounds}

Let the aggregate loss derived from the portfolio $\mathbf{X}$ be $\psi(\mathbf{X})$, for a function $\psi: \mathbb{R}^{n} \rightarrow$ $\mathbb{R}$, increasing in each coordinate and satisfying $\lim _{x_{i} \rightarrow+\infty} \psi(\mathbf{x})=+\infty$, for all $x_{1}, \ldots$, $x_{i-1}, x_{i+1}, \ldots, x_{n} \in \mathbb{R}$, and $i=1, \ldots, n$. We generalize the function $\phi: \mathbb{R}^{n-1} \rightarrow \mathbb{R}$ as follows:

$$
\phi\left(\mathbf{x}_{-n}\right):=\sum_{i=1}^{n-1} F_{i}\left(x_{i}\right)+F_{n}^{-}\left(\psi_{s}^{\sim}\left(\mathbf{x}_{-n}\right)\right)-n+1
$$


where $\psi_{s}^{\sim}: \mathbb{R}^{n-1} \rightarrow \mathbb{R}, \psi_{s}^{\sim}\left(\mathbf{x}_{-n}\right):=\sup \left\{x_{n} \in \mathbb{R}: \psi\left(\mathbf{x}_{-n}, x_{n}\right)<s\right\}$. Equation (3.2) is still valid within this extended framework:

$$
\mathbb{P}[\psi(\mathbf{X})<s] \geq \tau_{\psi}(s):=\sup _{\mathbf{x}_{-n} \in \mathbb{R}^{n-1}}\left[\phi\left(\mathbf{x}_{-n}\right)\right]^{+} .
$$

For a joint position $\psi(\mathbf{X})$ first order conditions are still necessary at the supremum defined in (A.1).

Theorem A.1. If $\psi_{s}^{\sim}$ is differentiable and, for a fixed real threshold $s, \tau_{\psi}(s)>p$, then $\tau_{\psi}(s)=\phi\left(\mathbf{x}_{-n}^{*}\right)$, where $\mathbf{x}_{-n}^{*}$ satisfies:

$$
f_{i}\left(x_{i}^{*}\right)+f_{n}\left(\psi_{s}^{\sim}\left(\mathbf{x}_{-n}^{*}\right)\right) \frac{\partial \psi_{s}^{\sim}}{\partial x_{i}}\left(\mathbf{x}_{-n}^{*}\right)=0, \quad i=1, \ldots, n-1 .
$$

Proof: The proof is analogous to that of Theorem 3.1. The conditions on $\psi$ are necessary to have that $\lim _{x_{i} \rightarrow+\infty} F_{n}^{-}\left(\psi_{s}^{\sim}\left(\left(\bar{x}_{F_{1}}, \ldots, \bar{x}_{F_{i-1}}, x_{i}, \bar{x}_{F_{i+1}}, \ldots, \bar{x}_{F_{n-1}}\right)\right)\right)=0$, for all $i=1, \ldots, n-1$.

Contrary to the case of the sum, conditions (A.2) may fail to be sufficient at the supremum in (A.1). General conditions yielding uniqueness of the vector $x_{-n}^{*}$ may be cumbersome to work with and will typically depend on the function $\psi$ and the fixed marginal densities. The GO algorithms described in Section 4.3 may therefore become useful already at this point, since they give also the location of the optimum function value. A condition analogous to (3.5) identifies the thresholds $s$ at which the standard bound can be calculated by using (A.2).

Lemma A.1. If $\psi$ is right-continuous in its last argument, $\tau_{\psi}(s)>p$ for every real threshold s satisfying

$$
s>\psi\left(F_{1}^{-1}\left(\frac{p+n-1}{n}\right), \ldots, F_{n}^{-1}\left(\frac{p+n-1}{n}\right)\right) .
$$

Proof: The proof is analogous to that of Lemma 3.1. Note that the assumption of continuity on $\psi$ avoids the case in which $\psi\left(\hat{\mathbf{x}}_{-n}, \psi_{s}^{\sim}\left(\hat{\mathbf{x}}_{-n}\right)\right)<s$, implying that $\psi_{s}^{\sim}\left(\hat{\mathbf{x}}_{-n}\right)=\hat{\mathbf{x}}_{-n}$ and $\tau_{\psi}(s)=p$.

\section{A.2 Dual bounds}

The dual result of Rüschendorf [1981] holds analogously for general measurable functionals $\psi$ :

$$
\begin{aligned}
\mathbb{P}[\psi(\mathbf{X})<s] \geq & 1-\inf \left\{\sum_{i=1}^{n} \int f_{i} d F_{i}: f_{i} \in \mathcal{B M}(\mathbb{R}), i \in N\right. \text { s.t. } \\
& \left.\sum_{i=1}^{n} f_{i}\left(x_{i}\right) \geq 1_{[s,+\infty)}(\psi(\mathbf{x})) \text { for all } \mathbf{x} \in \mathbb{R}^{n}\right\} .
\end{aligned}
$$


In order to find dual admissible functions for (A.4), we restrict our attention to functions of the form $\psi(\mathbf{x})=\sum_{i=1}^{n} h_{i}\left(x_{i}\right)$, for strictly increasing functions $h_{i}: \mathbb{R} \rightarrow \mathbb{R}, i \in N$. In this case, the computation of dual bounds is manageable with the same techniques as described in Section 4, but still relies on the calculation (analytical or numerical) of the vector $\mathbf{x}_{-n}^{*}$ attaining the standard bound.

Theorem A.2. For any function of the form $\psi(\mathbf{x})=\sum_{i=1}^{n} h_{i}\left(x_{i}\right)$, for strictly increasing, differentiable functions $h_{i}: \mathbb{R} \rightarrow \mathbb{R}, i \in N$, we have that

$$
\mathbb{P}[\psi(\mathbf{X})<s] \geq \delta_{\psi}(s):=1-\inf _{\mathbf{r} \in \prod_{i=1}^{n}\left(a_{i}, x_{i}^{*}\right)} \frac{\sum_{i=1}^{n} \int_{r_{i}}^{h_{i}^{-1}\left(s-\sum_{j \neq i} h_{j}\left(r_{j}\right)\right)}\left(1-F_{i}\left(x_{i}\right)\right) h_{i}^{\prime}\left(x_{i}\right) d x_{i}}{s-\sum_{i=1}^{n} h_{i}\left(r_{i}\right)},
$$

where $\mathbf{x}^{*}=\left(\mathbf{x}_{-n}^{*}, \psi_{s}^{\sim}\left(\mathbf{x}_{-n}^{*}\right)\right)$.

Proof: For an arbitrary vector $\mathbf{r} \in \prod_{i=1}^{n}\left(a_{i}, x_{i}^{*}\right)$ define $\hat{f}_{i}^{\mathbf{r}}: \mathbb{R} \rightarrow \mathbb{R}, i \in N$ as follows:

$$
\hat{f}_{i}^{\mathrm{r}}\left(x_{i}\right):= \begin{cases}0 & \text { if } x_{i} \leq r_{i}, \\ \frac{h_{i}\left(x_{i}\right)-h_{i}\left(r_{i}\right)}{s-\sum_{i=1}^{n} h_{i}\left(r_{i}\right)} & \text { if } r_{i}<x_{i} \leq h_{i}^{-1}\left(s-\sum_{j \neq i} h_{j}\left(r_{j}\right)\right), \\ 1 & \text { otherwise. }\end{cases}
$$

Analogously to the proof of Theorem 3.2, it is sufficient to check that $\hat{f}_{1}^{\mathbf{r}}, \ldots, \hat{f}_{n}^{\mathbf{r}}$ are an admissible dual choice in (A.4).

Acknowledgments The first author would like to thank EGRIE and the Geneva Association for the invitation to deliver the Geneva Risk Economics Lecture 2005 at the First World Risk and Insurance Economics Congress in Salt Lake City, 7-11 August, 2005. The lecture was partly based on the present paper. The authors also thank two anonymous referees for their helpful comments.

\section{References}

BASEL COMMITTEE ON BANKING SUPERVISION [2006]: International Convergence of Capital Measurement and Capital Standards. Basel: Bank for International Settlements.

BAZZARELLO, D., CRIELAARD, B., PIACENZA, F., and SOPRANO, A. [2006]: "Modeling Insurance Mitigation on Operational Risk Capital," Journal of Operational Risk, 1(1), 57-65.

CHAVEZ-DEMOULIN, V., EMBRECHTS, P., and NEŠLEHOVÁ, J. [2006]: "Quantitative Models for Operational Risk: Extremes, Dependence and Aggregation,” Journal of Banking and Finance, 30(10), 2635-2658.

De FONTNOUVELlE, P., DeJESUS-RUEFF, V., JORDAN, J., and ROSENGREN, E. [2005]: Capital and Risk: New Evidence on Implications of Large Operational Losses. Working Paper 03-5, Federal Reserve Bank of Boston.

DENUIT, M., GENEST, C., and MARCEAU, É. [1999]: "Stochastic Bounds on Sums of Dependent Risks," Insurance: Mathematics \& Economics, 25(1), 85-104.

EMBRECHTS, P., HÖING, A., and JURI, A. [2003]: "Using Copulae to Bound the Value-at-Risk for Functions of Dependent Risks," Finance and Stochastics, 7(2), 145-167.

EMBRECHTS, P., HÖING, A., and PUCCETTI, G. [2005]: "Worst VaR Scenarios,” Insurance: Mathematics \& Economics, 37(1), 115-134. 
EMBRECHTS, P., KLÜPPELBERG, C., and MIKOSCH, T. [1997]: Modelling Extremal Events, Berlin: Springer.

EMBRECHTS, P. and PUCCETTI, G. [2006]: "Bounds for Functions of Dependent Risks," Finance and Stochastics, 10(3), 341-352.

FOURER, R. [2000]: Nonlinear Programming Frequently Asked Questions. Available at http://wwwunix.mcs.anl.gov/otc/Guide/faq/nonlinear-programming-faq.html.

FRACHOT, A., MOUDOULAUD, O., and RONCALLI, T. [2004]: "Loss Distribution Approach in Practice," in The Basel Handbook: A Guide for Financial Practictioneers, M. K. Ong (Ed.), London: Risk Books, pp. 369-398.

HÜRLIMANN, W. [2004]: "Measuring Operational Risk Using a Mean Scaled Individual Risk Model," Applied Mathematics and Computation, 152(2), 425-447.

HUYER, W. and NEUMAIER, A. [1999]: "Global Optimization by Multilevel Coordinate Search," Journal of Global Optimization, 14(4), 331-355.

JONES, D.R., PERTTUNEN, C.D., and STUCKMAN, B.E. [1993]: "Lipschitzian Optimization Without the Lipschitz Constant," Journal of Optimization Theory and Application, 79(1), 157-181.

KÜHN, R. and NEU, P. [2004]: "Functional Correlation Approach to Operational Risk in Banking Organizations," Physica A, 322, 650-666.

LEIPPOLD, M. and VANINI, P. [2005]: “The Quantification of Operational Risk," Journal of Risk, 8(1), $59-85$.

McNEIL, A.J., FREY, R., and EMBRECHTS, P. [2005]: Quantitative Risk Management: Concepts, Techniques and Tools. Princeton: Princeton University Press.

MOSCADELLI, M. [2004]: "The Modelling of Operational Risk: Experience with the Analysis of the Data Collected by the Basel Committee." Temi di discussione, Banca d'Italia, URL: http://www.bancaditalia.it/ricerca/consultazioni/temidi/td04/td517/td_517/tema_517.pdf.

NEUMAIER, A. [2000]: Global Optimization Home Page. Available at http://www.mat.univie.ac.at/ $\sim$ neum/glopt.html.

NEUMAIER, A. [2004]: "Complete Search in Continuous Global Optimization and Constraint Satisfaction," Acta Numerica, 13, 271-369.

NEŠLEHOVÁ, J., EMBRECHTS, P., and CHAVEZ-DEMOULIN, V. [2006]: "Infinite-Mean Models and the LDA for Operational Risk," Journal of Operational Risk, 1(1), 3-25.

PEZIER, J. [1996]: "A Critique of Operational Risk Regulation and Control," in Operational Risk: Regulation, Analysis and Management, C. Alexander (Ed.), Hayward, CA: Financial Times Prentice Hall, Chapter 4.

PUCCETTI, G. [2005]: Dependency Bounds for Functions of Univariate and Multivariate Risks. Ph.D. thesis, University of Pisa. Second edition available at http://www.dmd.unifi.it/puccetti.

ROSENBERG, J. and SCHUERMANN, T. [2006]: "A General Approach to Integrated Risk Management with Skewed, Fat-Tailed Risks," Journal of Financial Economics, 79(3), 569-614.

RÜSCHENDORF, L. [1981]: "Sharpness of Fréchet-Bounds," Z. Wahrsch. Verw. Gebiete, 57(2), 293-302.

WILLIAMSON, R.C. and DOWNS, T. [1990]: "Probabilistic Arithmetic. I. Numerical Methods for Calculating Convolutions and Dependency Bounds," International Journal of Approximate Reasoning, 4(2), $89-158$. 